http://dx.doi.org/10.35381/r.k.v4i2.470

\title{
La contabilidad de gestión estratégica como herramienta multidisciplinar de planificación y control en la exportadora bananera Novamerc S.A.
}

Strategic management accounting as a multidisciplinary planning and control tool in the banana exporter Novamerc S.A.

\author{
Denisse Soraya Lindao Bravo \\ dlindaob@psg.ucacue.edu.ec \\ Universidad Católica de Cuenca, Cuenca \\ Ecuador: \\ https://orcid.org/0000-0002-8585-3867 \\ Cecilia Ivonne Narváez Zurita \\ inarvaez@ucacue.edu.ec \\ Universidad Católica de Cuenca, Cuenca \\ Ecuador: \\ https://orcid.org/0000-0002-7437-9880 \\ Juan Carlos Erazo Álvarez \\ jcerazo@ucacue.edu.ec \\ Universidad Católica de Cuenca, Cuenca \\ Ecuador \\ https://orcid.org/0000-0001-6480-2270
}

Recibido: 1 de septiembre de 2019

Aprobado: 30 de septiembre de 2019

\section{RESUMEN}

La situación económica actual del sector bananero exige buscar estrategias para obtener rentabilidad. Antiguamente, el margen de utilidad era determinado mediante el precio del producto menos costos y gastos; actualmente, se toma el margen de utilidad deseado con relación al precio del bien, es decir que depende de la gestión del administrador controlar los costos y gastos. La contabilidad de gestión estratégica va más allá de la contabilidad tradicional, raciona y proporciona información importante para la toma de decisiones. En este contexto, el presente artículo tiene por objetivo diseñar un sistema de contabilidad de gestión estratégica para la optimización de costos y gastos en la 
exportadora bananera Novamerc S.A. El sistema incluye herramientas de planificación y control, con miras a una gestión eficaz y eficiente de los recursos, como son el presupuesto, el modelo costeo objetivo, punto de equilibrio, entre otros.

Descriptores: Contabilidad de gestión estratégica; Costos de exportación; Sistemas de costeo; Herramientas financieras; Planificación y control.

\section{ABSTRACT}

The current economic situation of the banana sector requires seeking strategies to obtain profitability. In the past, the profit margin was determined by the price of the product minus costs and expenses; Currently, the desired profit margin is taken in relation to the price of the good, that is, it depends on the management of the administrator to control costs and expenses. Strategic management accounting goes beyond traditional accounting, rationing and providing important information for decision making. In this context, this article aims to design a strategic management accounting system for the optimization of costs and expenses in the banana exporter Novamerc S.A. The system includes planning and control tools, with a view to effective and efficient management of resources, such as the budget, the objective costing model, breakeven point, among others.

Descriptors: Strategic management accounting; Export costs; Costing systems; Financial tools; Planification and control.

\section{INTRODUCCIÓN}

La contabilidad ha experimentado un cambio considerable, ante la insuficiencia de información necesaria para la toma de decisiones que precisan los responsables de la gestión y control de la empresa, en tal sentido, se vio obligada a ampliar su campo de actuación hacia la obtención, elaboración y suministro de los datos originados en el proceso interno de la empresa, estableciendo estimaciones de futuro (Fullana y Paredes, 2008).

La contabilidad de gestión estratégica es un enfoque moderno de la gestión de costos, incluyendo las técnicas de evaluación de costos objetivo, el análisis funcional, la estimación de costos, las tablas de costos y el cálculo de costos por actividades. Como las empresas manejan estrategias particulares, precisan de diferentes sistemas de 
gestión de costos; en negocios donde los precios de los productos se rigen en base al mercado y sus demandas, la estrategia radica en convertirse en uno de los productores a menor costo, (Colina, 2017), entonces el sistema de gestión de costos debe diseñarse para ayudar a reducir costos y gastos; en cambio, si la estrategia busca diferenciación de los productos, el sistema de gestión de costos puede apoyar en la innovación y diseño (Tanaka, Yoshikawa, Innes y Mitchell, 1997).

Este estudio analiza el proceso de cambio estratégico en términos de estilo de gestión y de sistemas contables de gestión, para responder al problema ¿Cómo optimizar los costos y gastos en la exportadora bananera Novamerc S.A. de la ciudad de Machala? Por ello, el objetivo de la investigación es diseñar un sistema de contabilidad de gestión estratégica para la optimización de costos y gastos en la exportadora bananera Novamerc S.A.

\section{DESARROLLO}

\section{Contabilidad de gestión estratégica}

El mundo constantemente está evolucionando en todos los ámbitos, y en los negocios la tecnología y las estrategias competitivas son fundamentales para la sostenibilidad de las empresas a largo plazo. Tanaka et al. (1993), Prieto, Santidrián, y Aguilar, (2006), Ramírez (2013), Buelvas y Mejía (2014) y Laporta (2016) refieren que según el destino de la información la contabilidad se clasifica en contabilidad financiera y en contabilidad de gestión.

Las dos contabilidades se apoyan en el mismo sistema contable y se diferencian en los aspectos que se muestran en la tabla 1.

Diferencias entre contabilidad financiera y contabilidad de gestión.

Tabla 1.

Contabilidad financiera

Se enfoca en la preparación de estados financieros.

\section{Contabilidad de gestión}

Se enfoca en racionalizar información importante. 


\begin{tabular}{|ll|}
\hline Orientación global. & $\begin{array}{l}\text { En función de las diferentes etapas de la } \\
\text { cadena de valor. }\end{array}$ \\
\hline $\begin{array}{l}\text { Enfoque muy marcado hacia lo } \\
\text { interno. }\end{array}$ & $\begin{array}{l}\text { Enfoque muy marcado hacia lo externo } \\
\text { (mercado). }\end{array}$ \\
\hline Perspectiva ex post. & $\begin{array}{l}\text { Perspectiva ex ante y ex post. Oportunidad y } \\
\text { proyección. } \\
\text { Periodicida establecida por las } \\
\text { necesidades. } \\
\text { Suele tener una periodicidad anual. }\end{array}$ \\
$\begin{array}{l}\text { Dirigido a accionistas, instituciones al responsable de administrar. } \\
\text { de crédito y de control. }\end{array}$ & Necesidad de principios propios. \\
\hline Aplica PCGA y NIIF & Es opcional. \\
\hline Es obligatoria.
\end{tabular}

Fuente: Adaptado de Tanaka et al. (1993), Prieto et al. (2006) Ramírez (2013) y Laporta (2016).

La contabilidad de gestión estratégica enriquece la contabilidad tradicional, basada en la partida doble, argumentando que la contabilidad debe mirar mucho más allá y evaluar la posición competitiva de la empresa, para lo cual necesitará cooperación de otros departamentos que proporcionen información relativa a los mercados de la empresa y sus principales competidores, con énfasis en el largo plazo. La contabilidad de gestión estratégica se define como la entrega y análisis de información financiera, específicamente sobre la estructura de costos de la empresa, los costos de la competencia y su comportamiento en el tiempo; para ello, el rol de la contabilidad deberá extenderse con la finalidad de alinear los costos con la estrategia de la empresa, y para descubrir a breves rasgos la estructura de costos del competidor (Laporta, 2016).

Si el negocio se desenvuelve acorde al comportamiento del mercado, donde los precios son fijados por la competencia, la única alternativa es disminuir costos para mejorar la rentabilidad económica. Morillo (2001) refiere que se debe analizar la cadena de valor, el posicionamiento estratégico y las causales de costos, porque las reducciones de costo no solo deben darse cuando la empresa no esté obteniendo la utilidad deseada, sino encaminadas hacia la misión y visión de la empresa, mitigando las ineficiencias e incrementando a la vez la calidad en las actividades. 


\section{Sistemas de administración de costos}

Como se ha venido mencionando, la valuación de inventarios y costos de ventas es necesaria para la toma de decisiones, la misma que debe ser adecuada, confiable y puntual, especialmente cuando una empresa basa su estrategia en el liderazgo en costos. Existen varios sistemas de administración de costos desde la era de Revolución Industrial hasta la realidad actual de los negocios:

Sistemas de costeo basado en volúmenes: Cuevas (2001), Horngren, Sundem y Stratton (2006), Berrío y Castrillón (2008), Ramírez (2013) y Arredondo (2015) afirman que el sistema de costeo tradicional basado en volúmenes de producción prorratean los gastos indirectos de fabricación utilizando tasas como unidades producidas, horas-máquina $u$ horas-hombre, siendo los únicos costos que pueden agregarse, por lo que son válidos para efectos de contabilidad financiera, dejando otros costos de la cadena de valor.

Costeo basado en actividades ABC: Berrío y Castrillón (2008), Arredondo (2015), Toro (2016) y González, Narvéz, Lituma y Erazo (2019) sostienen que es una metodología que consiste en asignar costos a los insumos necesarios para ejecutar actividades de un proceso productivo. Una actividad es un trabajo que consume recursos de una empresa, e integra un proceso compuesto por varias tareas. Este sistema, según Cuervo, Osorio y Duque (2013) y Torres, Narváez, Juan y Ormaza (2019) es más apropiado para la toma de decisiones al incluir los gastos administrativos y de ventas, puesto que todos son recuperados con la venta y necesarios para que el producto llegue al cliente.

Costeo basado en metas (target costing): si se desea emprender un negocio, se empieza con el diseño del producto, lo que cuesta producir, y finalmente se fija el precio conforme al margen de utilidad esperado; sin embargo, el costeo basado en metas está dado en el escenario real de los negocios donde el mercado, sea nacional o internacional, y no los productores decide el precio de los bienes y servicios (Ramírez, 2013). Morillo (2001), Hansen y Mowen (2006), Horngren et al. (2006), Ramírez (2013) y Arredondo (2015) refieren que este sistema establece el costo máximo a incurrirse para poder vender un 
producto a un precio objetivo, siendo la única alternativa disminuir costos, como explotando los vínculos con los proveedores.

Es necesario el uso herramientas de apoyo para que los administradores realicen sus funciones de planeación y de control, como son el presupuesto y el punto de equilibrio.

\section{Presupuesto}

Hansen y Mowen (2006), Horngren et al. (2006), Laporta (2016) y Toro (2016) señalan que el presupuesto es una herramienta que cuantifica el plan de acción o proceso de producción, es decir los objetivos y las labores necesarias para lograrlo. Es necesario un control posterior comparando el plan original y estudiando los cambios. Para la elaboración del presupuesto debe seguirse el siguiente proceso:

Elección de periodos: trimestral, semestral y anual, divido por meses.

Organización: Una comisión de presupuestos formado por miembros de la alta gerencia, con las funciones de informar sobre la mecánica de la preparación del presupuesto, proporcionar los supuestos macro y microeconómicos, suministrar los datos del ejercicio o ejercicios pasados, hacer los cálculos necesarios y establecer tiempo de entrega de las propuestas.

Ejecución: la tarea de llevar a cabo el presupuesto es de todo el personal de la empresa. Control: seguimiento por parte de la comisión de presupuestos por cualquier situación y evitar que generen mayores problemas.

Manual de presupuesto: es un medio informativo que contiene los objetivos, las políticas, y asigna responsabilidades a todos y cada uno de los ejecutivos y obreros (Laporta, 2016).

\section{Punto de equilibrio}

Denominado más técnicamente modelo costo - volumen - utilidad (CVU), es una herramienta fundamental de planeación operativa a corto plazo. Laporta (2016) refiere que el punto de equilibrio o venta en equilibrio, es aquel nivel de actividad, expresado en 
términos de unidades vendidas, donde los ingresos totales se igualan a los costos totales; es decir, donde no se registran ni perdidas ni ganancias. El éxito del proceso de planeación a corto plazo depende de la creatividad e inteligencia con que se manejen las tres variables: costos, volúmenes y precios. Si la empresa quiere lograr una ventaja competitiva sustentable, como se revisó anteriormente, tiene tres caminos: liderazgo en costos, diferenciación o enfoque, y el modelo costo-volumen-utilidad dependerá de la estrategia que se adopte (Ramírez, 2013).

En el modelo CVU las ventas deben cubrir primero los costos variables, es decir aquellos íntimamente ligados al volumen de ventas. El resultado, llamado margen de contribución, representa la riqueza residual con la que la empresa intentará cubrir sus costos fijos:

Ventas

- Costos variables

= Margen de contribución

- Costos fijos

= Utilidad de operación

Existen dos formas de representar el punto de equilibrio:

- Algebraica

$$
x=\frac{C F}{P-V}
$$

Donde:

$\mathrm{P}=$ Precio por unidad

$X=$ Número de unidades a vender

$\mathrm{V}=$ Costo variable por unidad

$\mathrm{CF}=$ Costo fijo total

El punto de equilibrio se determina dividiendo los costos fijos totales entre el margen de contribución por unidad, que es el exceso entre el precio unitario respecto de los costos variables por unidad (Ramírez, 2013). En ocasiones los datos de precio unitario y costos variables no se encuentran disponibles, en estos casos, el cálculo del punto de equilibrio sustituirá el margen de contribución por unidad por el margen de contribución porcentual, 
de la siguiente forma:

(1) $M C \%=\frac{M C T}{I T} \quad$ ó

(2) $M C \%=\frac{P-V}{P}$

Aplicando este margen en la fórmula tendremos que:

(3) $P E=\frac{C F}{M C \%}$

Laporta (2016) señala que el punto de equilibrio de un solo producto puede darse en unidades y en valores. Para valores, propone la siguiente fórmula:

(4) $V e=\frac{C F}{r c}$

Donde:

$\mathrm{Ve}=$ Venta en equilibrio en valores

$\mathrm{CF}=$ Costos fijos

rc = Razón de costos

La razón de costos se determina con base a la razón de costos variables rcv:

(5) $\mathrm{rc}=1-\mathrm{rcV}$

(6) $\quad r c v=\frac{C V}{V}$

- Gráfica

Evalúa el efecto que sobre las utilidades tiene cualquier movimiento o cambio de costos, volumen de ventas y precios. En el gráfico el eje horizontal representa las ventas en unidades, y el vertical, los costos totales, calculados en diferentes niveles de venta. El punto de intersección de ambas rectas representa el punto de equilibrio (Laporta, 2016). 
Representación del punto de equilibrio.

Figura 1.

\section{Punto de equilibrio}

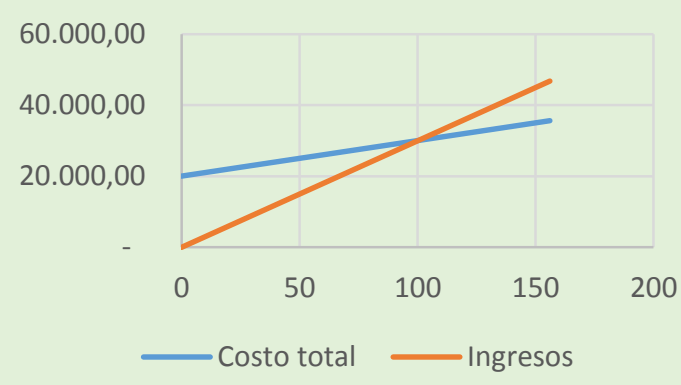

Fuente: (Ramírez, 2013).

ProMéxico (2010) afirma que el punto de equilibrio de un solo producto no es común, porque la mayoría de las empresas produce o comercializa distintos productos o líneas de productos. El problema consiste en qué proporción de los costos fijos se le atribuirá a cada producto o línea de productos, con base de un pronóstico de ventas. Para este prorrateo existen varias opciones:

a) Identificar una unidad de medida que integre los productos o líneas de productos.

b) Contribución del producto o línea de productos a la rentabilidad de la empresa, en volumen y precios.

Para el cálculo se utiliza el método de la contribución marginal:

(7) $\quad \mathrm{MT}=(\mathrm{P}-\mathrm{CV}) \times \mathrm{VOL}$

Donde:

MT = Contribución Marginal Total

$\mathrm{P}=$ Precio de venta

$\mathrm{CV}=$ Costo variable unitario

$\mathrm{VOL}=$ Volumen de ventas

\section{Costos de exportación}

Además de los costos de producción, la exportación incluye gastos propios del proceso de exportación pre-embarque, embarque y post-embarque, tales como embalaje, carga, 
envío al puerto, trámites de aduana, seguro de transporte, transporte internacional y otros gastos en el país de destino que generalmente son cubiertos por el importador. Los términos de venta incoterms, acordados entre el importador y el exportador, determinan los derechos y las obligaciones de los mismos. Su elección también se asienta sobre prácticas comerciales usuales, características del producto y la experiencia adquirida. Son trece términos comerciales, elaborados por la Cámara de Comercio Internacional (CCI), última versión del 2010, ordenados en cuatro categorías por responsabilidades:

Incoterms.

Tabla 2.

\begin{tabular}{|llll|}
\hline Grupo & Sigla & \multicolumn{1}{c|}{ Inglés } & \multicolumn{1}{c|}{ Español } \\
\hline E & EXW & Ex Works & En fábrica \\
\hline F & FCA & Free Carrier & Lugar convenido \\
& FAS & Free Alongside Ship & Franco al costado del buque \\
& FOB & Free on Board & Franco a bordo \\
\hline C & CFR & Cost and Freight & Costo y Flete \\
& CIF & Cost, Insurance and Freight & Costo, Seguro y Flete \\
& CPT & Carriage paid to & Transporte pagado hasta \\
& CIP & Carriage and Insurrance paid to & Transporte y seguro pagado hasta \\
\hline D & DAF & Delivered at Frontier & Entregado en Frontera \\
& DES & Delivered ex ship & Entregado sobre Buque \\
& DEQ & Delivered Ex Quay & Entregado en Muelle \\
& DDU & Delivered Duty Unpaid & Entregado, derechos no pagados \\
& DDP & Delivery Duty Paid & Entregado, derechos pagados \\
\hline
\end{tabular}

Fuente: Adaptado de (Ministerio de Desarrollo Económico de Buenos Aires, 2002).

Los términos Franco a Bordo (FOB) y Costo, Seguro y Flete (CIF) son los más utilizados en el comercio internacional, así como por las entidades gubernamentales como plataforma de estudio para ponderar sus estadísticas sobre balanzas comerciales, volúmenes y precios de exportación, políticas arancelarias y para-arancelarias, entre otros. En el FOB el vendedor cumple con su obligación de entrega cuando la mercancía, despachada para exportación, ha sobrepasado la borda del buque en el puerto de 
embarque convenido; a partir de este momento el comprador asume todos los costos y riesgos de la mercancía. En el CIF el vendedor cumple con su obligación de entrega cuando la mercancía sobrepasa la borda del buque en el puerto de embarque convenido, asumiendo el mismo el pago de los costos, el flete para hacer llegar la mercancía al puerto de destino convenido, la contratación del seguro y el pago de la prima correspondiente.

\section{METODOLOGÍA}

El diseño de la presente investigación fue no experimental, ya que se analizaron las variables en su estado natural, sin realizar ningún proceso de manipulación. La investigación se realizó bajo el enfoque mixto, es decir utilizando las fortalezas de la investigación cualitativa en la primera etapa, donde se examinó literatura sobre contabilidad de gestión estratégica, costos de producción y costos de exportación; y cuantitativa, en la segunda etapa, mediante un diagnóstico a la empresa objeto de estudio. El alcance fue descriptivo por cuanto se fundamentó teóricamente y luego se especificó el comportamiento de las variables de estudio con los datos obtenidos de las encuestas y entrevistas a un grupo de personas que laboran en la empresa Novamerc S.A. La finalidad del estudio fue transversal ya que el levantamiento de información fue aplicado en un solo momento del tiempo.

Los métodos empleados fueron el inductivo - deductivo, de lo general a lo particular y en sentido contrario; el analítico - sintético que descompuso el objeto de estudio en cada una de sus partes, y luego se integró esas partes para estudiarlas de manera holística e integral; el histórico - lógico que proporcionó un sistema de evaluación y síntesis de pruebas sistematizadas que permitieron entender los hechos que justifican el estado actual de las variables; $y$, el sistémico, que ejecutó un proceso para el logro de objetivos mediante la propuesta. Se utilizó las técnicas de análisis documental, observación, encuesta y entrevista, mediante los instrumentos: ficha de análisis documental, guía de observación, cuestionario y guía de preguntas. 
La población o universo de la investigación estuvo conformado por: 1 directivo, 3 personas del área contable y liquidaciones, 1 asistente de logística y 1 persona encargada de inventarios, por consiguiente, la población ascendió a 6 personas para la aplicación de la encuesta, y más la entrevista realizada a la gerente general de Novamerc S.A. Al ser una población reducida, no se procedió con el cálculo muestral.

\section{RESULTADOS}

Entre los principales resultados que se obtuvieron mediante los métodos, técnicas e instrumentos de investigación, constan los siguientes:

Volumen de cajas: Los empleados conocen cuántas cajas semanalmente exporta la empresa, dando como resultado que actualmente se exporta un promedio de 22570 cajas de banano semanales.

Costos de exportación: acorde a los resultados se concluye que se tiene restringido el acceso a los registros de contabilidad de costos, archivos digitales y demás información confidencial, a personal ajeno a estas funciones.

Cotizaciones: como buena práctica la empresa recibe varias cotizaciones, con el fin de escoger la mejor propuesta para las adquisiciones de bienes y servicios; sin embargo, en lo que respecta a la fruta, se maneja empíricamente el proceso de compra.

Recepción de inventarios: la empresa aplica control interno de comparar las cantidades, precios y condiciones de la factura contra las cotizaciones $u$ órdenes de compra; no obstante, las verificaciones de volúmenes se torna superficial al recibir gran cantidad de materiales, lo que hace difícil cuantificarlos.

Control de inventarios: se evidenció la inexistencia de políticas y procedimientos adecuados para la administración de los inventarios, en especial en cuanto a verificación de cantidades, especificaciones técnicas, el desecho, venta o control sobre desperdicios o mermas, y a la restricción del acceso a la bodega a personal ajeno a estas funciones. Tomas físicas de inventario: la empresa realiza periódicamente tomas físicas de inventarios; sin embargo, solo se practica una o dos veces al año, en ocasión de la 
auditoría de estados financieros.

Competencia: La competencia tiene costos y gastos inferiores a los de Novamerc, puesto que solo la administración eficiente de los mismos, permitirá incrementar la rentabilidad de la empresa.

Tecnología: el sistema de contabilidad instaurado no cuenta con un módulo de costos que proporcione información en tiempo real para la toma de decisiones.

Estrategias: existen escazas estrategias operativas con relación a la optimización de costos y gastos que permitan cumplir con la misión, visión y objetivos institucionales.

Transacciones: las adquisiciones de bienes se realizan por escrito, en cuanto a la compra de banano se maneja informalmente y a través de intermediarios.

Comprobantes de venta: los documentos de venta son válidos y se registran oportunamente; cabe mencionar que, se encontraron facturas registradas como gastos no deducibles, ya que no han sido entregadas a tiempo al departamento de contabilidad; sin embargo, no son relevantes.

Registros: la empresa cuenta con Manual de Políticas contables que son requeridos por motivo de auditoría externa a los estados financieros, exigidos por los organismos de control. Dicho manual sirve de apoyo para el manejo de cuentas y una guía de contabilización.

Pagos: a) no se revisa previo al pago final al proveedor, los anticipos entregados por la empresa y los descuentos otorgados previamente por el proveedor; b) no se respetan plazos establecidos para el pago a proveedores, existen facturas vencidas por más de 90 días; c) existen pagos que son aprobados en ausencia de la persona encargada, aumentando el riesgo en la empresa.

Archivo: la documentación del año en curso y del anterior se encuentra archivada en las oficinas de la empresa. La de años anteriores, se archiva en bodega para su conservación y mantenimiento, con base en las disposiciones técnicas y jurídicas vigentes. La información digital se encuentra respaldada en un disco duro externo.

A partir de los resultados expuestos se propone las herramientas de planificación: 


\section{Propuesta.}

Figura 2.

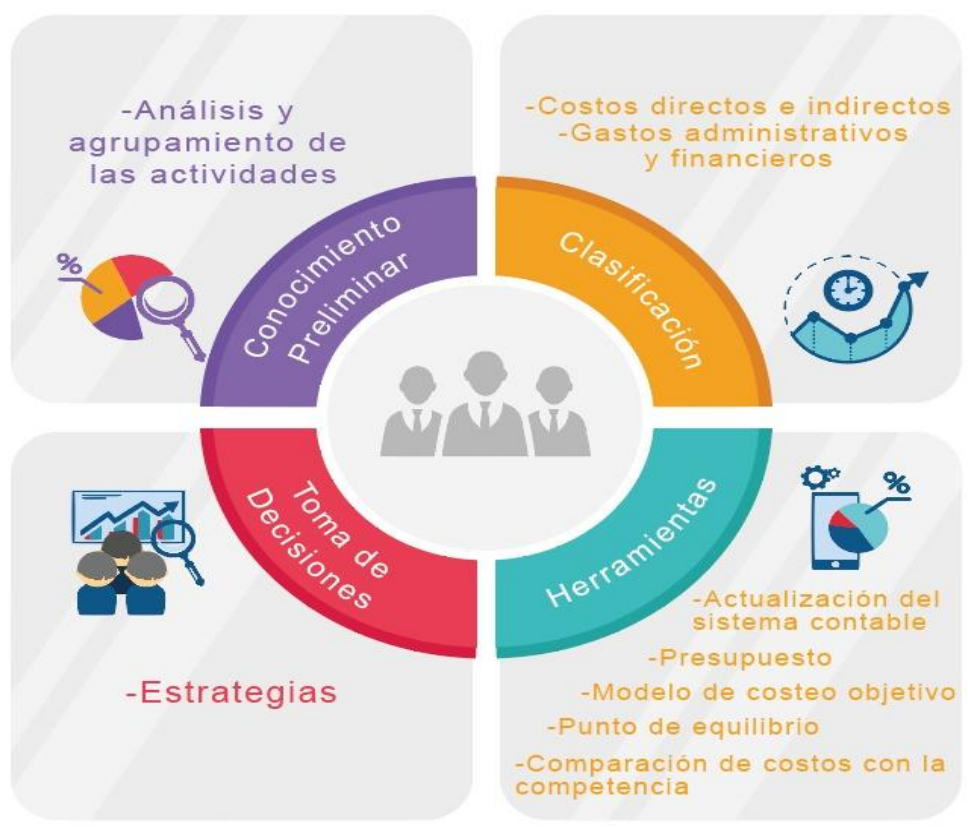

Análisis y agrupamiento de las actividades: para el agrupamiento de las actividades es necesario identificar primero los elementos del costo: materia prima, materiales y recursos humanos que se consumen para la exportación semanal de bananos. Los elementos del costo y las actividades identificadas en el proceso de exportación de Novamerc S.A. se detallan en las tablas 3 y 4 :

\section{Elementos del costo de exportación.}

\section{Tabla 3.}

Codificación

C1

C2

C3

C4

C5

C6
Elementos del costo

Fruta

Cartón

Material de empaque

Estiba, paletizado y puerto

Dctos. y trámites exportación

Consecución de fruta

Identificación de actividades. 
Tabla 4.

\begin{tabular}{|c|c|}
\hline $\begin{array}{l}\text { Procedimiento } \\
\text { CM }\end{array}$ & $\begin{array}{l}\text { Actividades } \\
\text { Comercialización }\end{array}$ \\
\hline CM.1 & $\begin{array}{l}\text { Contacto y negocio con los clientes fijos a finales de año cuántas } \\
\text { cajas de fruta se procesará semanalmente el próximo año. }\end{array}$ \\
\hline CM.2 & $\begin{array}{l}\text { Solicitud a las Agencias Navieras generen el Booking (reservas de } \\
\text { espacios asignados en el Buque) según el destino de la fruta, ya } \\
\text { sea bajo cubierta o contenedores. }\end{array}$ \\
\hline CM.3 & $\begin{array}{l}\text { Establecimiento del estimado de embarque: marca, puerto, vapor, } \\
\text { cantidad, peso y la Autorización Única de Control Previo (AUCP). }\end{array}$ \\
\hline OP & Operaciones \\
\hline OP.1 & $\begin{array}{l}\text { Comunicación a los intermediarios la reserva de cupo de cada } \\
\text { marca para la consecución de fruta. }\end{array}$ \\
\hline OP.2 & $\begin{array}{l}\text { Contactar al productor y definir cuántas cajas van a procesar en la } \\
\text { semana. Comunicación al personal competente las cajas a } \\
\text { procesarse para la exportación. }\end{array}$ \\
\hline OP.3 & $\begin{array}{l}\text { Planificación estimada semanal de las operaciones, estableciendo } \\
\text { el orden de corte para los productores. }\end{array}$ \\
\hline OP.4 & $\begin{array}{l}\text { Coordinación de la entrega de materiales e insumos a los } \\
\text { productores. }\end{array}$ \\
\hline CP & Compras \\
\hline CP.1 & Búsqueda de proveedores \\
\hline CP.2 & Elección y definición de los criterios de selección del proveedor \\
\hline CP.3 & Adquisición de cartón y material de empaque \\
\hline CP.4 & Cumplimiento en calidad, volumen y precio del cartón y material. \\
\hline $\begin{array}{l}\text { OP } \\
\text { OP.5 }\end{array}$ & $\begin{array}{l}\text { Operaciones } \\
\text { Entrega de cartón y materiales a los productores }\end{array}$ \\
\hline OP.6 & Inspecciones de Contenedores en Depósitos \\
\hline OP.7 & Transporte de contenedores \\
\hline OP.8 & Consolidación de carga e inspección de calidad \\
\hline OP.9 & Colocación de termógrafo y cierre del contenedor \\
\hline EX & Exportaciones \\
\hline EX.1 & $\begin{array}{l}\text { Documentación para el Ingreso a Puerto: Documento emitido para } \\
\text { las Autorizaciones de Ingreso y Salida de Vehículos en Puerto } \\
\text { (AISV) y Declaración Aduanera de Exportación (DAE) }\end{array}$ \\
\hline EX.2 & Inspección de antinarcóticos previa notificación \\
\hline
\end{tabular}




\begin{tabular}{ll}
\hline EX.3 & $\begin{array}{l}\text { Actualización de cantidades de cajas con las navieras para el cierre } \\
\text { de cada reserva }\end{array}$ \\
EX.4 & Emisión y aprobación de Bill of Lading $(B L)$ \\
EX.5 & Emisión de factura y cierre de DAE y AUCP \\
EX.6 & Emisión de certificados fitosanitario y de origen \\
EX.7 & Envío de certificados y BL originales al cliente vía courier
\end{tabular}

\section{Centro de costos}

Novamerc S.A. exporta bananos de producción tradicional y de producción orgánica, y de varias marcas, por lo que es menester agrupar la contabilidad por centro de costos:

\section{Centros de costos.}

\section{Tabla 5.}

\begin{tabular}{cll}
\hline Codificación & Producción & Marca \\
\hline EX & Tradicional & Excelban \\
DM & Tradicional & Del Monte \\
FF & Orgánica & Fyffes \\
DO & Orgánica & Del Monte \\
\hline
\end{tabular}

\section{Clasificación de costos directos e indirectos}

Al tener identificados los costos y las actividades, es momento de clasificar los costos en directos e indirectos, y por marcas.

\section{Clasificación de costos marca Excelban.}

\section{Tabla 6.}

\begin{tabular}{cll}
\hline Cod. & Clasificación & Componentes \\
\hline C1 & Directo & Banano \\
C2 & Directo & Cartón corrugado \\
C3 & Directo & $\begin{array}{l}\text { Fundas, etiquetas, ligas, fungaflor, mertec, alumbre y sulfato de } \\
\text { aluminio }\end{array}$ \\
C4 & Indirecto & $\begin{array}{l}\text { Emisión de BL, transferencia de carga banano directo a buque, } \\
\text { porteo de banano semipaletizado, inspección Agrocalidad, } \\
\text { enmienda de transmisión electrónica, auditoria de conteo de } \\
\text { cajas, colocación de termógrafos, cajas de banano bajadas en } \\
\text { bodega, camiones verificación de fruta en bodega. }\end{array}$ \\
\hline
\end{tabular}




\begin{tabular}{|c|c|c|}
\hline C5 & Indirecto & $\begin{array}{l}\text { Envíos de documentos al exterior, honorarios agente afianzado, } \\
\text { trámite certificado fitosanitario y de origen. }\end{array}$ \\
\hline C6 & Indirecto & Comisiones a intermediarios \\
\hline \multicolumn{3}{|c|}{$\begin{array}{l}\text { Clasificación de costos marca Del Monte tradicional. } \\
\text { Tabla } 7 .\end{array}$} \\
\hline Cod. & Clasificación & Componentes \\
\hline C1 & Directo & Banano \\
\hline C2 & Directo & Cartón corrugado \\
\hline C3 & Directo & $\begin{array}{l}\text { Fundas, etiquetas, alumbre, papel absorvente, strata y rally, } \\
\text { pallets, esquineros } 24 \mathrm{~cm} \text {, esquineros } 1.50 \mathrm{~cm} \text {, grapas, zunchos }\end{array}$ \\
\hline C4 & Indirecto & $\begin{array}{l}\text { Emisión de BL, servicio de supervisión portuaria, transferencia } \\
\text { de carga de banano paletizado directo a buque, inspección } \\
\text { antinarcóticos, inspección pre embarque, arribo tardío al } \\
\text { terminal, inspección de la calidad }\end{array}$ \\
\hline C5 & Indirecto & Honorarios agente afianzado, trámite certificado fitosanitario \\
\hline C6 & Indirecto & Comisiones a intermediarios \\
\hline
\end{tabular}

\section{Clasificación de costos marca Fyffes.}

\section{Tabla 8.}

\begin{tabular}{lll}
\hline Cod. & Clasificación & Componentes \\
\hline C1 & Directo & Banano \\
C2 & Directo & Cartón corrugado \\
C3 & Directo & $\begin{array}{l}\text { Fundas, etiquetas, alumbre 2, liga, BC 1000, pallets, esquineros } \\
23 \mathrm{~cm} \text {, esquineros } 220 \mathrm{~cm} \text {, grapas, zunchos }\end{array}$ \\
C4 & Indirecto & $\begin{array}{l}\text { Servicio de supervisión portuaria, servicio de trasbordo, } \\
\text { inspección de condición BASC, recepción y porteo de } \\
\text { contenedores, certificación VGM, cortina de bloqueo, sellos, } \\
\text { inspección antinarcóticos, transporte de contenedores, } \\
\text { evaluación de calidad, filtros y termógrafos }\end{array}$ \\
C5 & Indirecto & $\begin{array}{l}\text { Honorarios agente afianzado, trámite certificado fitosanitario y de } \\
\text { origen y certificados de transacción }\end{array}$ \\
C6 & Indirecto & \begin{tabular}{l} 
Comisiones a intermediarios \\
\hline
\end{tabular} \\
\hline
\end{tabular}

Clasificación de costos marca Del Monte orgánica.

Tabla 9. 


\begin{tabular}{|c|c|c|}
\hline Cod. & Clasificación & Componentes \\
\hline C1 & Directo & Banano \\
\hline $\mathrm{C} 2$ & Directo & Cartón corrugado \\
\hline C3 & Directo & $\begin{array}{l}\text { Fundas, cintas, etiquetas, alumbre } 2 \text {, liga, BC } 1000 \text {, papel } \\
\text { absorvente, paraseal, pallets, esquineros } 24 \mathrm{~cm} \text {, esquineros } \\
196 \mathrm{~cm} \text {, grapas, zunchos }\end{array}$ \\
\hline C4 & Indirecto & $\begin{array}{l}\text { Emisión de BL, sellos, recepción, manipuleo, conexión y } \\
\text { desconexión de contenedores, certificación VGM, arribo tardío } \\
\text { al terminal, servicio de trasbordo, paletizado y logística, } \\
\text { consolidación de cajas, sellamiento de contenedores, } \\
\text { inspección antinarcóticos, inspección de condición BASC, } \\
\text { transporte de contenedores, evaluación de calidad, filtros, } \\
\text { sellos y bolsas de seguridad }\end{array}$ \\
\hline C5 & Indirecto & $\begin{array}{l}\text { Honorarios agente afianzado, trámite certificado fitosanitario y } \\
\text { certificados de transacción }\end{array}$ \\
\hline C6 & Indirecto & Comisiones a intermediarios \\
\hline
\end{tabular}

\section{Actualización del sistema contable}

Es necesaria la actualización del sistema contable que proporcione información financiera y de gestión en tiempo real. Se basó en el Software Contable Administrativo Integral scai versión 2018 que actualmente está operativo en la empresa y en base a los requerimientos de sus usuarios. 
Novamerc S.A

Propuesta de sistema contable

\begin{tabular}{|c|c|}
\hline Módulos & Procesos \\
\hline \multirow{7}{*}{ Inventarios } & Archivo maestro de artículos \\
\hline & Archivo maestro de proveedores \\
\hline & Archivo de productores \\
\hline & Embarques semanales \\
\hline & Registrar ingresos a bodega \\
\hline & Grabar egresos \\
\hline & Transferencias entre bodegas \\
\hline \multirow{7}{*}{ Liquidaciones } & Archivo de productores \\
\hline & Embarques semanales \\
\hline & Rubros de liquidaciones \\
\hline & Imprimir liquidaciones individual y por lote \\
\hline & Generar comprobantes de pago \\
\hline & Ingresar datos de puerto \\
\hline & Liquidación de alcance para ajustar precios \\
\hline \multirow{6}{*}{ Rol de pagos } & Registro de anticipos \\
\hline & Calcular rol mensual \\
\hline & Imprimir rol de pagos mensual \\
\hline & Imprimir rol de pagos individual \\
\hline & Autorización para transferencias \\
\hline & Impresión de cheques \\
\hline \multirow{7}{*}{ Contabilidad } & Grabar / corregir cuentas contables \\
\hline & Comprobantes contables \\
\hline & Enlazar facturas con ingresos a bodega \\
\hline & Revisar comprobantes descuadrados \\
\hline & Revisar e imprimir Libro diario \\
\hline & Cierres mensuales \\
\hline & Impuestos \\
\hline Caja / Bancos & Conciliar documentos bancarios \\
\hline \multirow{4}{*}{ Costos } & Hojas de costos \\
\hline & Parametrización de materiales \\
\hline & Parametrización de mano de obra \\
\hline & Parametrización de otros costos \\
\hline \multirow{3}{*}{ Facturación } & Archivo de clientes \\
\hline & Grabar facturas \\
\hline & Grabar notas de crédito \\
\hline \multirow{2}{*}{ Cuentas por cobrar } & Abonos/ Cancelaciones de clientes \\
\hline & Imprimir recibos de pago \\
\hline \multirow{3}{*}{ Cuentas por pagar } & Anticipos a proveedores \\
\hline & Abonos/Cancelaciones a proveedores \\
\hline & Imprimir comprobantes de pago \\
\hline \multirow{4}{*}{ Inversiones } & Registrar datos de propiedad, planta y equipo \\
\hline & Registrar datos de activos de inversión \\
\hline & Calcular depreciación de un activo \\
\hline & Depreciar grupo de activos \\
\hline
\end{tabular}




\section{Novamerc S.A. \\ Propuesta de sistema contable}

\begin{tabular}{|c|c|}
\hline Módulo & Requerimientos \\
\hline \multirow{7}{*}{ Inventarios } & Clasificado por línea, componente y marca \\
\hline & Razón social, RUC, dirección y correo \\
\hline & Clasificado por zona, por grupo y por intermediario \\
\hline & Marca, semana, vapor, precio y alícuota de cartón y material \\
\hline & Tipo de movimiento, proveedor, embarque, bodega \\
\hline & Tipo de movimiento, productor, embarque, cajas, bodega \\
\hline & Multibodega \\
\hline \multirow{10}{*}{ Liquidaciones } & Clasificado por zona, por grupo y por intermediario \\
\hline & Marca, semana, vapor, precio y alícuota de cartón y material \\
\hline & Descuentos y pagos adicionales a productores \\
\hline & Generar liquidaciones por embarques semanales \\
\hline & Cartón descontado por componentes: tapa, fondo y cartulina \\
\hline & Visualización automática de préstamos o anticipos \\
\hline & Impresión de liquidaciones por embarque o escoger un productor \\
\hline & Sistema de Pago Interbancario (SPI) por embarque y carga a cada productor \\
\hline & Registro de cajas por embarques y por productor \\
\hline & Precio de contrato, precio spot y descuentos adicionales \\
\hline \multirow{2}{*}{ Rol de pagos } & Anticipos individual y por lote \\
\hline & Visualización automática de préstamos o anticipos \\
\hline \multirow{10}{*}{ Contabilidad } & Nivel 5 \\
\hline & Automatización de registros contables \\
\hline & Registro de facturas de compras por marca y semana \\
\hline & Registro de notas de crédito y documento modificado \\
\hline & Registro de facturas soportes de reembolsos de gastos \\
\hline & $\begin{array}{l}\text { Vincular los registros contables de las facturas de compras de inventarios con los } \\
\text { ingresos a bodega }\end{array}$ \\
\hline & Vincular el registro de la factura de compra de banano a las liquidaciones de fruta \\
\hline & Generar Anexo Transaccional Simplificado \\
\hline & Generar Anexo Transaccional Simplificado ATS \\
\hline & Corregir datos de ATS \\
\hline \multirow{3}{*}{ Caja / Bancos } & Control de cheques \\
\hline & Control de débitos \\
\hline & Control de transferencias \\
\hline \multirow{5}{*}{ Costos } & Centro de costos por marca \\
\hline & Proyección de costos según estadísticas \\
\hline & Materiales directos e indirectos por embarques \\
\hline & Mano de obra directa e indirecta por embarques \\
\hline & Costos indirectos o imprevistos por embarques \\
\hline \multirow{4}{*}{ Facturación } & Razón social, identificación, dirección y correo \\
\hline & Facturas de exportaciones, ventas locales y reembolsos \\
\hline & Cálculo automático de peso neto y bruto de las exportaciones \\
\hline & Importar datos de refrendos de exportaciones al ATS \\
\hline Inversiones & $\begin{array}{l}\text { Código, descripción, marca, modelo, serie, fecha de compra, valor y \% de depreciación } \\
\text { Depreciación mensual, trimestral, anual }\end{array}$ \\
\hline
\end{tabular}




\section{Presupuesto}

Cajas Excelban: el presupuesto para el año 2020 se ha basado en las variaciones de los años 2014 - 2015 - 2016 - 2017 - 2018 - 2019 tanto en cantidades de cajas como en precios de la fruta en el mercado internacional. Para el año 2019 que aún no ha concluido, se tomó el histórico de semana 1 a 32, y para las semanas 33 a 52, el promedio porcentual de los años 2014 a 2018 de $-15 \%$ en cantidad y $12 \%$ en precio de la fruta. Pronosticado el periodo 2019, se procedió a establecer un nuevo promedio porcentual de los años 2014 a 2019 dando como resultado -12\% en cantidad de cajas y manteniéndose el incremento de $12 \%$ en el precio de fruta, con estos datos se proyectó el año 2020.

En cuanto a precios de fruta a productores, se tomó el promedio porcentual de los años 2015 a 2019, dando como resultado un incremento del 9\%.

Cajas Del Monte: el presupuesto de ventas del año 2020 se ha basado en el contrato del año 2019, ya que no tendría mayor variación de los 7 contenedores que se exportan actualmente. En cuanto a precios de fruta, se ha revisado la variación porcentual de semanas 1 a del año 2018 y 2019, al no contar con más datos históricos. Para la caja Del Monte tradicional se calculó un incremento del 1\% del precio de la caja de banano, el mismo que fue aplicado también en las compras; $y$, para la orgánica, una disminución del $-5 \%$ tanto en exportación como en la compra de banano a productores.

Cajas Fyffes: a partir de semana 19 del año 2019 se está exportando este tipo de caja, por lo que en el presupuesto del año 2020 se está considerando 2 contenedores de 960 cajas y al mismo precio de US \$ 11.80 en exportaciones, y US \$ 8.15 en compras.

El total de cajas de todas las marcas y tipo proyectadas para el periodo 2020 son 925735 , lo que da un promedio de 17803 cajas semanales.

Para los costos y gastos de exportación y los gastos de administración y financieros, se tomó la variación de los años 2017 - 2018 y 2019, dando como resultado un incremento del $2 \%$. Cabe mencionar que en el presupuesto de gastos operacionales se considera el cartón y estiba de las cajas excelban, sin embargo, éstos son asumidos y cancelados a 
Revista Arbitrada Interdisciplinaria KOINONIA

Año IV. Vol IV. N². Edición Especial 2019

Hecho el depósito de Ley: FA2016000010 ISSN: $2542-3088$

FUNDACIÓN KOINONIA (F.K). Santa Ana de Coro. Venezuela.

Henry Patricio Llivicura Ávila; Juan Carlos Erazo Álvarez; Cecilia Ivonne Narváez Zurita

los proveedores directamente por el importador, motivo por el cual son restados en el estado de resultados proyectado año 2020. 
Revista Arbitrada Interdisciplinaria KOINONIA

Año IV. Vol IV. N². Edición Especial 2019

Hecho el depósito de Ley: FA2016000010

ISSN: 2542-3088

FUNDACIÓN KOINONIA (F.K). Santa Ana de Coro. Venezuela.

Henry Patricio Llivicura Ávila; Juan Carlos Erazo Álvarez; Cecilia Ivonne Narváez Zurita

Novamerc S.A.

Estado de Resultados Proyectado

Año 2020

\begin{tabular}{|c|c|c|c|c|c|c|}
\hline & Denominación & EX & DM & FF & DO & Total \\
\hline & Cantidad cajas & 476455 & 149760 & 99840 & 199680 & 925735 \\
\hline \multicolumn{6}{|c|}{ Ingresos por exportaciones } & \multirow[t]{3}{*}{$8,264,602.01$} \\
\hline & Cajas tradicionales & $3,750,535.19$ & $1,231,327.62$ & & & \\
\hline & Cajas orgánicas & & & $1,178,112.00$ & $2,104,627.20$ & \\
\hline \multicolumn{3}{|c|}{ Costos directos e indirectos } & & & & \multirow[t]{7}{*}{$7,726,452.53$} \\
\hline C1 & Fruta & $3,236,758.26$ & $913,536.00$ & $813,696.00$ & $1,557,504.00$ & \\
\hline $\mathrm{C} 2$ & Cartón & - & $207,747.07$ & $149,700.10$ & $276,996.10$ & \\
\hline C3 & Material de empaque & $68,037.74$ & $24,440.83$ & $70,267.39$ & $124,240.90$ & \\
\hline C4 & Estiba, paletizado y puerto & $34,685.91$ & $47,354.11$ & $72,304.13$ & $69,249.02$ & \\
\hline C5 & Dctos. y trámites exportaciór & $4,859.84$ & $1,527.55$ & $4,073.47$ & $6,289.92$ & \\
\hline C6 & Consecución de fruta & $34,018.87$ & $3,055.10$ & $2,036.74$ & $4,073.47$ & \\
\hline & Utilidad bruta & $372,174.56$ & $33,666.95$ & $66,034.18$ & $66,273.79$ & $538,149.48$ \\
\hline \multicolumn{3}{|c|}{ Gastos administrativos y financieros } & & & & \multirow[t]{19}{*}{$615,484.38$} \\
\hline & Sueldos y beneficios sociale؛ & $123,201.91$ & $38,725.01$ & $25,816.68$ & $51,633.35$ & \\
\hline & Remuneraciones a otros & $35,412.67$ & $11,130.97$ & $7,420.64$ & $14,841.29$ & \\
\hline & Mantenimientos y reparacion & $6,060.83$ & $1,905.05$ & $1,270.03$ & $2,540.07$ & \\
\hline & Arriendos & $5,091.48$ & $1,600.36$ & $1,066.91$ & $2,133.82$ & \\
\hline & Combustibles y lubricantes & $2,907.33$ & 913.84 & 609.22 & $1,218.45$ & \\
\hline & Seguros & $8,190.10$ & $2,574.32$ & $1,716.22$ & $3,432.43$ & \\
\hline & Transporte y courier & $2,565.39$ & 806.36 & 537.57 & $1,075.14$ & \\
\hline & Gastos de gestión & $10,888.46$ & $3,422.48$ & $2,281.65$ & $4,563.30$ & \\
\hline & Gastos de viaje & $1,938.09$ & 609.18 & 406.12 & 812.25 & \\
\hline & Promoción y publicidad & $2,013.48$ & 632.88 & 421.92 & 843.84 & \\
\hline & Agua, energía y telecomunicí & $8,870.13$ & $2,788.07$ & $1,858.71$ & $3,717.43$ & \\
\hline & Notarios y registradores & $1,822.75$ & 572.93 & 381.95 & 763.91 & \\
\hline & Impuestos, contribuciones y & $16,675.40$ & $5,241.44$ & $3,494.29$ & $6,988.58$ & \\
\hline & Depreciaciones y amortizaci & $15,222.59$ & $4,784.79$ & $3,189.86$ & $6,379.72$ & \\
\hline & Suministros y materiales & $1,889.32$ & 593.85 & 395.90 & 791.81 & \\
\hline & Afiliación y cuotas gremiales & $3,115.64$ & 979.31 & 652.88 & $1,305.75$ & \\
\hline & Comisiones bancarias & $1,622.71$ & 510.05 & 340.04 & 680.07 & \\
\hline & Intereses & $69,287.61$ & $21,778.59$ & $14,519.06$ & $29,038.12$ & \\
\hline & Utilidad/pérdida operaciona & $55,398.65$ & $-65,902.54$ & -345.48 & $-66,485.53$ & $-77,334.90$ \\
\hline & Impuesto a la renta único & $58,889.81$ & $18,510.34$ & $12,340.22$ & $24,680.45$ & $114,420.82$ \\
\hline & Utilidad/pérdida neta & $-3,491.16$ & $-84,412.88$ & $-12,685.71$ & $-91,165.98$ & $-191,755.72$ \\
\hline
\end{tabular}




\section{Novamerc S.A.}

Estado de Resultados Proyectado por caja

Año 2020

\begin{tabular}{|l|r|r|r|r|}
\hline \multicolumn{1}{|c|}{ Denominación } & EX & DM & FF & \multicolumn{1}{|c|}{ DO } \\
\hline Ingresos por exportaciones & 7.87 & 8.22 & 11.80 & 10.54 \\
\hline Costos de exportación & $\mathbf{8 . 6 2}$ & $\mathbf{8 . 0 0}$ & $\mathbf{1 1 . 1 4}$ & $\mathbf{1 0 . 2 1}$ \\
\hline Fruta & 6.79 & 6.10 & 8.15 & 7.80 \\
\hline Cartón & 1.38 & 1.39 & 1.50 & 1.39 \\
\hline Material de empaque & 0.14 & 0.16 & 0.70 & 0.62 \\
\hline Estiba, paletizado y puerto & 0.22 & 0.32 & 0.72 & 0.35 \\
\hline Dctos. y trámites exportación & 0.01 & 0.01 & 0.04 & 0.03 \\
\hline Consecución de fruta & 0.07 & 0.02 & 0.02 & 0.02 \\
\hline Utilidad bruta & $\mathbf{0 . 7 8}$ & $\mathbf{0 . 2 2}$ & $\mathbf{0 . 6 6}$ & $\mathbf{0 . 3 3}$ \\
\hline Gastos administrativos y & $\mathbf{0 . 6 6}$ & $\mathbf{0 . 6 6}$ & $\mathbf{0 . 6 6}$ & $\mathbf{0 . 6 6}$ \\
\hline Sueldos y beneficios sociales & 0.26 & 0.26 & 0.26 & 0.26 \\
\hline Remuneraciones a otros & 0.07 & 0.07 & 0.07 & 0.07 \\
\hline Mantenimientos y reparaciones & 0.01 & 0.01 & 0.01 & 0.01 \\
\hline Arriendos & 0.01 & 0.01 & 0.01 & 0.01 \\
\hline Combustibles y lubricantes & 0.01 & 0.01 & 0.01 & 0.01 \\
\hline Seguros & 0.02 & 0.02 & 0.02 & 0.02 \\
\hline Transporte y courier & 0.01 & 0.01 & 0.01 & 0.01 \\
\hline Gastos de gestión & 0.02 & 0.02 & 0.02 & 0.02 \\
\hline Gastos de viaje & 0.00 & 0.00 & 0.00 & 0.00 \\
\hline Promoción y publicidad & 0.00 & 0.00 & 0.00 & 0.00 \\
\hline Agua, energía y telecomunicac & 0.02 & 0.02 & 0.02 & 0.02 \\
\hline Notarios y registradores & 0.00 & 0.00 & 0.00 & 0.00 \\
\hline Impuestos, contribuciones y otı & 0.03 & 0.03 & 0.03 & 0.03 \\
\hline Depreciaciones y amortizacion & 0.03 & 0.03 & 0.03 & 0.03 \\
\hline Suministros y materiales & 0.00 & 0.00 & 0.00 & 0.00 \\
\hline Afiliación y cuotas gremiales & 0.01 & 0.01 & 0.01 & 0.01 \\
\hline Comisiones bancarias & 0.00 & 0.00 & 0.00 & 0.00 \\
\hline Intereses & 0.15 & 0.15 & 0.15 & 0.15 \\
\hline Total gastos operativos & $\mathbf{9 . 2 8}$ & $\mathbf{8 . 6 6}$ & $\mathbf{1 1 . 8 0}$ & $\mathbf{1 0 . 8 7}$ \\
\hline Utilidad/pérdida operacional & $\mathbf{0 . 1 2}$ & $-\mathbf{0 . 4 4}$ & $-\mathbf{0 . 0 0}$ & $-\mathbf{0 . 3 3}$ \\
\hline Impuesto a la renta único & 0.12 & 0.12 & 0.12 & 0.12 \\
\hline Utilida/pérdida neta & $-\mathbf{0 . 0 1}$ & $-\mathbf{0 . 5 6}$ & $-\mathbf{0 . 1 3}$ & $-\mathbf{0 . 4 6}$ \\
\hline & & & & \\
\hline
\end{tabular}




\section{Costeo objetivo}

El comercio de banano es extremadamente competitivo, lo que hace que los márgenes unitarios sean bajos. Al no poder fijar un precio, se elaboró en base a los precios históricos. En cuanto a la utilidad deseada, la alta gerencia determinó que sea 0.10 por caja de banano de producción tradicional, y 0.15 por caja de banano orgánico. El costo meta máximo por caja Excelban es $\$ 7.39$, Del Monte convencional $\$ 8.12$, Fyffes orgánicas $\$ 11.65$ y Del Monte orgánica $\$ 10.39$ como se muestra a continuación:

Novamerc S.A.

Modelo de costeo objetivo

Año 2020

\begin{tabular}{|c|c|r|r|r|}
\hline Tipo & Marca & \multicolumn{3}{|c|}{ Costo meta $=$ Precio de venta - Margen de utilidad deseada } \\
\hline \multirow{2}{*}{ Tradicionales } & EX & 7.39 & 7.49 & 0.10 \\
\cline { 2 - 5 } & DM & 8.12 & 8.22 & 0.10 \\
\hline \multirow{2}{*}{ Orgánicas } & FF & 11.65 & 11.80 & 0.15 \\
\cline { 2 - 5 } & DO & 10.39 & 10.54 & 0.15 \\
\hline
\end{tabular}

\section{Punto de equilibrio}

En base a las proyecciones realizadas, el punto de equilibrio es de 1'729.474 cajas al año, lo que significaría un promedio de 33259 cajas semanales para que en la exportadora bananera Novamerc S.A. los ingresos sean iguales a los costos; por lo tanto, a partir de 33260 cajas semanales se estaría empezando a generar utilidades, mientras que la venta de 33258 o de un número menor de cajas semanales significaría pérdidas.

$\mathrm{PE}=\mathrm{CF} / \mathrm{MCP}$

$P E=615.484,38 / 0,36$

$\mathrm{PE}=11^{\prime} 729.474$ 


\begin{tabular}{|l|r|r|r|r|}
\hline \multicolumn{1}{|c|}{ Denominación } & \multicolumn{1}{c|}{ EX } & \multicolumn{1}{c|}{ DM } & \multicolumn{1}{c|}{ FF } & \multicolumn{1}{c|}{ D } \\
\hline PVU & 7.49 & 8.22 & 11.80 & 10.54 \\
\hline CVU & 7.03 & 8.12 & 11.26 & 10.33 \\
\hline Margen de contribución & 0.46 & 0.10 & 0.54 & 0.21 \\
\hline Porcentaje de participación & $51 \%$ & $16 \%$ & $11 \%$ & $22 \%$ \\
\hline Margen de contribución ponde & 0.24 & 0.02 & 0.06 & 0.04 \\
\hline
\end{tabular}

\begin{tabular}{|c|c|r|r|r|r|r|r|r|r|r|}
\hline \multirow{2}{*}{ Tipo } & Marca & $\begin{array}{c}\text { PE } \\
\text { Unidades }\end{array}$ & $\begin{array}{c}\text { Porcentaje } \\
\text { de } \\
\text { participación }\end{array}$ & $\begin{array}{c}\text { Unidades por } \\
\text { producto }\end{array}$ & \multicolumn{2}{|c|}{ PVU } & \multicolumn{2}{|c|}{ CVU } & \multicolumn{2}{|c|}{$\begin{array}{c}\text { Margen de } \\
\text { contribución }\end{array}$} \\
\hline \multirow{2}{*}{ Tradicionales } & EX & 1729474 & $51 \%$ & 890,121 & 7.49 & $6,664,268.90$ & 7.03 & $6,255,117.76$ & 0.46 & $409,151.14$ \\
\cline { 2 - 12 } & DM & 1729474 & $16 \%$ & 279,784 & 8.22 & $2,300,387.84$ & 8.12 & $2,272,071.99$ & 0.10 & $28,315.85$ \\
\hline \multirow{2}{*}{ Orgánicas } & FF & 1729474 & $11 \%$ & 186,523 & 11.80 & $2,200,969.49$ & 11.26 & $2,100,657.50$ & 0.54 & $100,311.98$ \\
\cline { 2 - 11 } & DO & 1729474 & $22 \%$ & 373,046 & 10.54 & $3,931,901.42$ & 10.33 & $3,854,196.01$ & 0.21 & $77,705.41$ \\
\hline & Promedio & 33259 & Total & $1,729,474$ & Total & $15,097,527.64$ & Total & $14,482,043.26$ & Total & $615,484.38$ \\
\hline
\end{tabular}

En base a estas herramientas multidisciplinares de planificación y control para la exportadora Novamerc S.A., se propone implementar las siguientes estrategias:

\section{a. Economía de escala o capacidad instalada}

Las empresas son capaces de generar beneficios gracias a las grandes cantidades de banano que comercializan, lo cual reduce el costo unitario de la caja. Además, el volumen otorga a la empresa los recursos económicos para invertir en innovaciones tecnológicas como maquinaria para agilizar procesos y evitar tener que contratar más mano de obra. b. Página web y redes sociales

Publicar contenido educativo y no puramente comercial, aumentará la visibilidad del negocio; además es necesario interactuar con los usuarios.

c. Testimonios, confianza y credibilidad

Para llegar a más productores de banano, se puede entrevistar a algunos productores antiguos que den testimonio sobre el compromiso de la empresa con respecto a los precios pactados, capacitaciones y la atención recibida, esto generará confianza y credibilidad; dichos testimonios incluirían fotos y se publicarían digitalmente en la web y 
en la empresa.

d. Alianzas

Buscar alianzas estratégicas con asociaciones de pequeños productores y con intermediarios o referidos que recomienden a la exportadora con otros clientes del exterior, con la finalidad de llegar a más personas del sector.

e. Responsabilidad Social Empresarial (RSE)

La RSE añade valor a la empresa, beneficia a la comunidad y respeta el medio ambiente, es por eso necesario dar atención a los grupos de interés o stakeholders como son clientes, proveedores y trabajadores en general.

Si se logran implementar estas estrategias, el presupuesto para el año 2020, en base a los históricos, ya no estaría en pérdida como se demostró anteriormente. 
Revista Arbitrada Interdisciplinaria KOINONIA

Año IV. Vol IV. ํo2. Edición Especial 2019

Hecho el depósito de Ley: FA2016000010

ISSN: 2542-3088

FUNDACIÓN KOINONIA (F.K). Santa Ana de Coro. Venezuela.

Henry Patricio Llivicura Ávila; Juan Carlos Erazo Álvarez; Cecilia Ivonne Narváez Zurita

Novamerc S.A.

Estado de Resultados Proyectado con estrategias

Año 2020

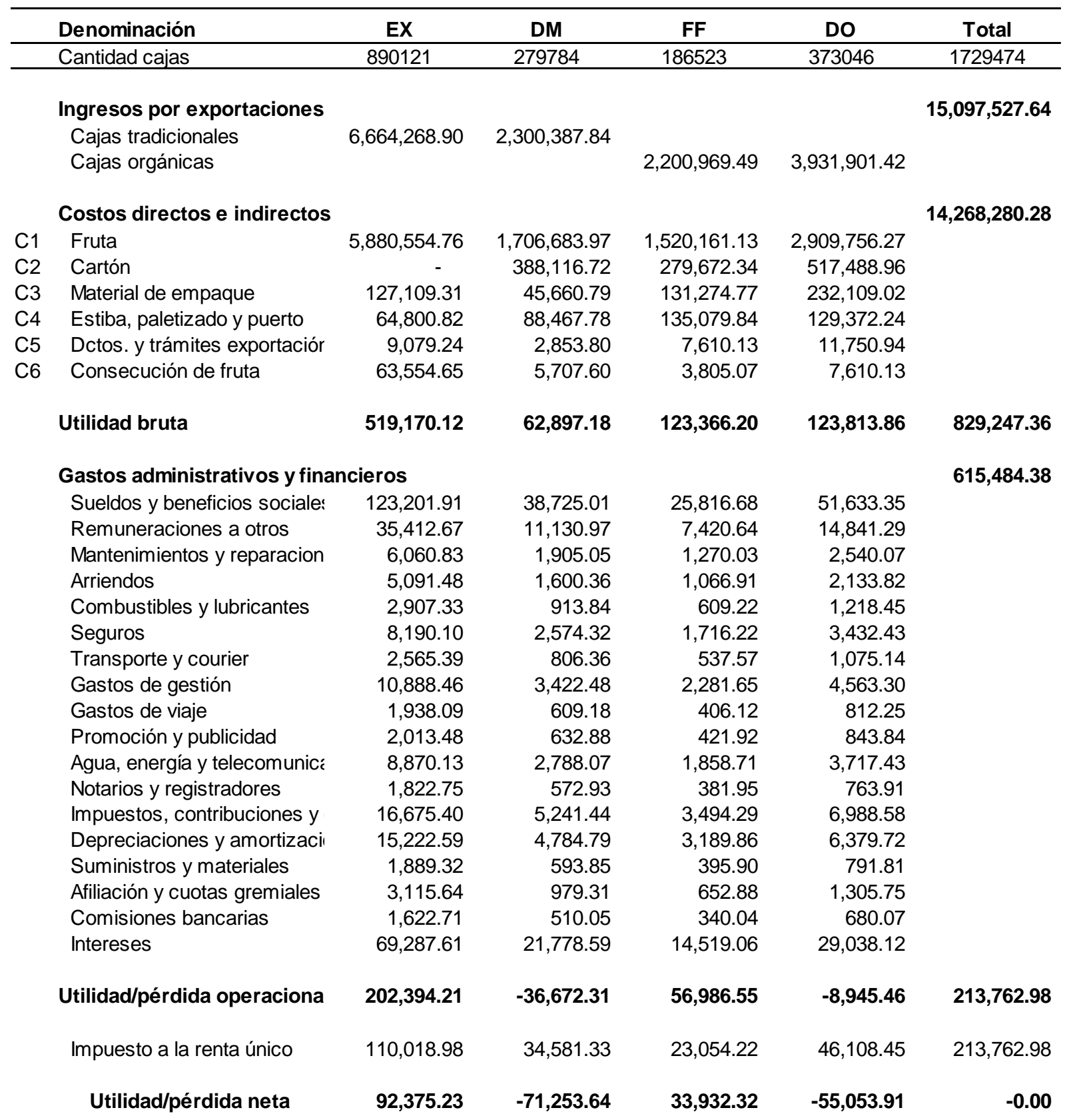




\section{DISCUSIÓN}

1. La contabilidad de gestión estratégica enriquece la contabilidad tradicional, permitiendo evaluar la posición competitiva de la empresa, para lo cual necesitará cooperación de otros departamentos que proporcionen información relativa a los mercados de la empresa y sus principales competidores.

2. La valuación de inventarios y costos de ventas es necesaria para la toma de decisiones, por lo que la empresa debe escoger el sistema que más se adapte a su situación particular, con el fin de obtener información adecuada y confiable, especialmente si basa su estrategia en el liderazgo en costos.

3. El presupuesto y el punto de equilibrio son herramientas fundamentales para el plan de acción a corto plazo, para conocer los objetivos y las labores necesarias para lograrlo. Es necesario un control posterior e ir registrando los cambios con respecto al plan original y analizando las desviaciones en la sucesión de tareas.

4. Las empresas manejan estrategias particulares: en negocios donde los precios de los productos se rigen en base al mercado y sus demandas, la estrategia radica en convertirse en uno de los productores a menor costo; para otros negocios la estrategia busca diferenciación de los productos a través de la innovación y diseño.

\section{REFERENCIAS CONSULTADAS}

1. Arredondo, M. (2015). Accounting and cost analysis. Mexico: Grupo Editorial Patria.

2. Berrío, D., \& Castrillón, J. (2008). Costs to manage manufacturing, commercial and service organizations. Barranquilla: Ediciones Uninorte.

3. Buelvas, C., \& Mejía, G. (2014). The role of management accounting in the accounting information system and its impact on the profitability of companies. Panorama económico, 91-108. 
4. Cuervo, J., Osorio, J., \& Duque, M. (2013). Activity Based Costing ABC. Bogotá: Ecoe Ediciones.

5. Cuesta, U. (2012). Strategic Planning and Creativity. Madrid: ESIC.

6. Cuevas, C. (2001). Cost accounting. Management and management approach. Bogota: Pearson Educación de Colombia Ltda.

7. Fullana, C., \& Paredes, J. (2008). Cost Accounting Manual. Madrid: Delta, Publicaciones Universitarias.

8. González, H., Narvéz, C., Lituma, M., \& Erazo, J. (2019). ABC / ABM cost management system for the textile industry. Vatex company case. Visionario Digital, 23.

9. Hansen, D., \& Mowen, M. (2006).

10. Cost management. Accounting and control. Mexico: Cengage Learning Editores, S.A.

11. Colina, M. (2017). Ambiente virtual de aprendizaje de contabilidad ii para las horas de trabajo independiente del programa nacional de formación en administración. Revista Arbitrada Interdisciplinaria Koinonía, 2(3), 92-108. Recuperado de http://fundacionkoinonia.com.ve/ojs/index.php/revistakoinonia/article/view/54/41

12. Horngren, C., Sundem, G., \& Stratton, W. (2006). Administrative accounting. Mexico: Pearson Educación.

13. Laporta, R. (2016). Costs and business management. Bogota: Ecoe Ediciones.

14. Ministerio de Desarrollo Económico de Buenos Aires. (23 de 12 de 2002). Guide for the first export. Dirección de Industrias Creativas y Comercio Exterior. Obtenido de http://primeraexportacion.com.ar/documentos-tecnicos/el-precio-deexportacion/

15. Morillo, M. (2001). Financial Profitability and Cost Reduction. Actualidad Contable Faces.

16. Prieto, B., Santidrián, A., \& Aguilar, P. (2006). Cost and management accounting. Madrid: Delta Publicaciones. 
17. ProMéxico. (03 de 2010). How to determine the export price. Obtained from http://www.promexico.gob.mx/documentos/pdf/ComoDeterminarEIPrecioDeExpor tacion.pdf

18. Ramírez, D. (2013). Administrative Accounting. A strategic approach to compete. Monterrey: Mc Graw Hill, Interamericana Editores S.A.

19. Tanaka, M., Yoshikawa, T., Innes, J., \& Mitchell, F. (1993). Modern Cost Management. Madrid: Ediciones Díaz de Santos, S.A.

20. Toro, F. (2016). Costs ABC and budgets Bogota: Ecoe Ediciones Ltda.

21.Torres, M., Narváez, C., Juan, E., \& Ormaza, J. (2019). Costs ABC/ABM as a business management tool. Company case PlanERP Cía. Ltda. Visionario Digital, 21.

22. Torres, Z. (2014). Strategic management. México: Grupo Editorial Patria S.A.

\section{REFERENCES CONSULTED}

1. Arredondo, M. (2015). Accounting and cost analysis. Mexico: Patria Editorial Group.

2. Berrío, D., \& Castrillón, J. (2008). Costs to manage manufacturing, commercial and service organizations. Barranquilla: Uninorte editions.

3. Buelvas, C., \& Mejía, G. (2014). The role of management accounting in the accounting information system and its impact on the profitability of companies. Economic outlook, 91-108.

4. Cuervo, J., Osorio, J., \& Duque, M. (2013). Activity Based Costing ABC. Bogotá: Ecoe Editions.

5. Cuesta, U. (2012). Strategic Planning and Creativity. Madrid: ESIC.

6. Cuevas, C. (2001). Cost accounting Management and management approach. Bogota: Pearson Educación de Colombia Ltda.

7. Fullana, C., \& Paredes, J. (2008). Cost Accounting Manual. Madrid: Delta, University Publications. 
8. González, H., Narvéz, C., Lituma, M., \& Erazo, J. (2019). ABC / ABM cost management system for the textile industry. Vatex company case. Digital Visionary, 23.

9. Hansen, D., \& Mowen, M. (2006).

10. Cost management. Accounting and control. Mexico: Cengage Learning Editores, S.A.

11. Colina, M. (2017). Virtual accounting learning environment ii for working hours independent of the national administration training program. Interdisciplinary Arbitrated Review Koinonía, 2 (3), 92-108. Recovered from http://fundacionkoinonia.com.ve/ojs/index.php/revistakoinonia/article/view/54/41

12. Horngren, C., Sundem, G., \& Stratton, W. (2006). Administrative accounting Mexico: Pearson Education.

13. Laporta, R. (2016). Costs and business management. Bogota: Ecoe Editions.

14. Ministry of Economic Development of Buenos Aires. (23 of 12 of 2002). Guide for the first export. Directorate of Creative Industries and Foreign Trade. Retrieved from http://primeraexportacion.com.ar/documentos-tecnicos/el-precionadeexportacion/

15. Morillo, M. (2001). Financial Profitability and Cost Reduction. Current Accounting Faces.

16. Prieto, B., Santidrián, A., \& Aguilar, P. (2006). Cost and management accounting. Madrid: Delta Publications.

17. ProMéxico. (03 of 2010). How to determine the export price. Obtained from http://www.promexico.gob.mx/documentos/pdf/ComoDeterminarEIPrecioDeExpor tacion.pdf

18. Ramírez, D. (2013). Administrative Accounting A strategic approach to compete. Monterrey: Mc Graw Hill, Interamericana Editores S.A.

19. Tanaka, M., Yoshikawa, T., Innes, J., \& Mitchell, F. (1993). Modern Cost Management Madrid: Ediciones Díaz de Santos, S.A.

20. Toro, F. (2016). Costs ABC and budgets Bogota: Ecoe Ediciones Ltda. 
Revista Arbitrada Interdisciplinaria KOINONIA

Año IV. Vol IV. N². Edición Especial 2019

Hecho el depósito de Ley: FA2016000010

ISSN: 2542-3088

FUNDACIÓN KOINONIA (F.K). Santa Ana de Coro. Venezuela.

Henry Patricio Llivicura Ávila; Juan Carlos Erazo Álvarez; Cecilia Ivonne Narváez Zurita

21.Torres, M., Narváez, C., Juan, E., \& Ormaza, J. (2019). Costs ABC / ABM as a business management tool. Company case PlanERP Cía. Ltda. Digital Visionary, 21.

22. Torres, Z. (2014). Strategic management Mexico: Grupo Editorial Patria S.A.

(C2019 por el autor. Este artículo es de acceso abierto y distribuido según los términos y condiciones de la licencia Creative Commons Atribución-NoComercial-Compartirlgual 4.0 Internacional (CC BY-NC-SA 4.0)

(https://creativecommons.org/licenses/by-nc-sa/4.0/). 